\title{
Weyl-Invariant Extension of the Metric-Affine Gravity
}

\author{
R. Vazirian, ${ }^{1}$ M. R. Tanhayi, ${ }^{2}$ and Z. A. Motahar $^{3}$ \\ ${ }^{1}$ Plasma Physics Research Center, Islamic Azad University, Science and Research Branch, Tehran 1477893855, Iran \\ ${ }^{2}$ Department of Physics, Islamic Azad University, Central Tehran Branch, Tehran 8683114676, Iran \\ ${ }^{3}$ Department of Physics, University of Malaya, 50603 Kuala Lumpur, Malaysia
}

Correspondence should be addressed to M. R. Tanhayi; m-tanhayi@iauctb.ac.ir

Received 30 September 2014; Accepted 28 November 2014

Academic Editor: Anastasios Petkou

Copyright (C) 2015 R. Vazirian et al. This is an open access article distributed under the Creative Commons Attribution License, which permits unrestricted use, distribution, and reproduction in any medium, provided the original work is properly cited. The publication of this article was funded by $\mathrm{SCOAP}^{3}$.

Metric-affine geometry provides a nontrivial extension of the general relativity where the metric and connection are treated as the two independent fundamental quantities in constructing the spacetime (with nonvanishing torsion and nonmetricity). In this paper, we study the generic form of action in this formalism and then construct the Weyl-invariant version of this theory. It is shown that, in Weitzenböck space, the obtained Weyl-invariant action can cover the conformally invariant teleparallel action. Finally, the related field equations are obtained in the general case.

\section{Introduction}

Extended theories of gravity have become a field of interest in recent years due to the lack of a theory which could fully describe gravity as one of the fundamental interactions together with other ones. Shortcoming of the current gravitation theory in cosmological scales, despite all the successes in solar system tests, is another reason for developing the idea of extending it and has confirmed the need to surpass Einstein's general theory of relativity (GR) $[1-3]$. The extension can be made in different ways such as geometrical, dynamical, and higher dimensions or even a combination of them $[4,5]$. In this paper, we will focus on geometric extension to gravity under the notion of metric-affine formalism.

Our current description of gravity is based, via theory of GR, on Riemannian geometry in which metric is the only geometrical object needed to determine the spacetime structure and connection is considered to be metric connection, the well-known Christoffel symbols. In such a context, as $a$ priori, the connection is symmetric, that is, torsion-free condition, and is also assumed to be compatible with the metric $\left(\nabla_{\lambda} g_{\mu \nu}=0\right)$. To enlarge this scheme, one can set the two presumptions aside and think of metric-affine geometry in which metric and connection are independent geometrical quantities. Therefore, connection is no longer compatible with the metric and torsion-free condition is relaxed; thus, in addition to Christoffel symbols, the affine connection would contain an antisymmetric part and nonmetric terms as well. The transition from Riemann to metric-affine geometry has led to metric-affine gravity (MAG), an extended theory of gravity which has been extensively studied during recent decades from different viewpoints, and a variety of nonRiemannian cosmology models are proposed based on it (e.g., see [6-11]).

The underlying idea of GR that explains gravity in terms of geometric properties of spacetime remains unaffected in MAG and just new concepts are added to this picture by introducing the torsion and nonmetricity tensors in description of gravity. Almost all of the spacetime geometries such as Riemann-Cartan, Weyl, and Minkowski geometries can be obtained by constraining the three aforementioned tensors $[12,13]$; this is one of the peculiarities of metric-affine formalism.

In this paper, we study the conformally invariant metricaffine theory. The conformal theories of gravity are of great importance; for example, in the framework of quantum gravity it is proved that such theories are better to renormalize [14] and also, from the phenomenological point of view, it is shown that the solutions of conformal invariant theories (e.g., Weyl gravity) can explain the extraordinary speed of rotation 
curves of galaxies and also they can address the cosmological constant problem $[15,16]$. Thus, considering the conformal invariance within the context of MAG seems to be worthwhile, which has been studied in some papers [17, 18]. (There are other choices of the conformal transformation of the torsion tensor which are studied in [19-22].) We first consider the action in its general format in MAG and then fix the degrees of freedom by imposing the conformal invariance.

The organization of the paper is as follows. The conventions and general aspects of metric-affine geometry are briefly reviewed in Section 2. In Section 3, the most general second order action in metric-affine formalism is presented in terms of free parameters. Weyl-invariant version of the action is investigated in Section 4 by determining the free parameters and the subject will be concluded in the fifth section. The field equations of the general action are presented in the Appendix.

\section{Metric-Affine Geometry}

As mentioned, the main idea in MAG is the independence of metric and connection, both of which are being the fundamental quantities indicating the spacetime structure and carry their own dynamics in contrast with GR where metric is the only independent dynamical variable [23, 24]. Note that, in Palatini formalism of GR, such an assumption holds, but it brings nothing novel to the theory. In presence of the gravitational field, spacetime can be curved or twisted and these features of spacetime are characterized by curvature and torsion tensors defined in terms of the affine connection and its derivatives as

$$
\mathbf{R}_{\beta \mu \nu}^{\lambda}=-\partial_{\nu} \Gamma_{\beta \mu}^{\lambda}+\partial_{\mu} \Gamma_{\beta \nu}^{\lambda}+\Gamma_{\alpha \mu}^{\lambda} \Gamma_{\beta \nu}^{\alpha}-\Gamma_{\alpha \nu}^{\lambda} \Gamma_{\beta \mu}^{\alpha}
$$

and (square brackets and parentheses in relations show antisymmetrization and symmetrization over indices, respectively)

$$
S_{\mu \nu}^{\alpha} \equiv \Gamma_{[\mu \nu]}^{\alpha}=\frac{\Gamma_{\mu \nu}^{\alpha}-\Gamma_{\nu \mu}^{\alpha}}{2} .
$$

These two definitions are obtained from the covariant derivatives commutator acting on a vector field,

$$
\left[\nabla_{\mu}, \nabla_{\nu}\right] V^{\lambda}=R_{\beta \mu \nu}^{\lambda} V^{\beta}+2 S_{\mu \nu}^{\alpha} \nabla_{\alpha} V^{\lambda},
$$

where $\nabla$ denotes covariant derivative associated with the affine connection and for a tensor field is defined as

$$
\nabla_{\mu} A_{\nu}^{\lambda}=\partial_{\mu} A_{\nu}^{\lambda}+\Gamma_{\beta \mu}^{\lambda} A_{\nu}^{\beta}-\Gamma_{\nu \mu}^{\beta} A_{\beta}^{\lambda}
$$

It must be emphasized that the third index of the connection is conventionally chosen to be in the direction along which differentiation is done (the order of indices must be carefully considered since the connection coefficients are supposed not to be completely symmetric with nonvanishing torsion tensor) $[23,25]$.

Nonmetricity tensor is the other geometrical object defined as

$$
\nabla_{\lambda} g_{\mu \nu} \equiv Q_{\lambda \mu \nu}
$$

from which it is implied that inner product of vectors and so their length and the angle between them are not conserved when parallel transported along a curve in spacetime $[4,26]$.

Another difference that arose in this setup is the increase of dynamical degrees of freedom. Noting that the affine connection is independent of the metric and is not constrained in general, it thus contains 64 independent components in fourdimensional spacetime. As it is obvious by definitions given above, torsion and nonmetricity are rank-three tensors being antisymmetric in first and symmetric in last pair of indices, respectively. Due to these symmetry properties, the two quantities will constitute the 64 independent components of the affine connection (24 components of torsion tensor and 40 components of nonmetricity tensor). Thereby, the overall number of the independent components will become 74 by taking into account the 10 independent components of the metric, a symmetric second rank tensor, together with the connection.

It is straightforward to show that the affine connection can be expressed in the following form:

$$
\Gamma_{\mu \nu}^{\lambda}=\left\{\begin{array}{c}
\lambda \\
\mu \nu
\end{array}\right\}+K_{\mu \nu}^{\lambda}+L_{\mu \nu}^{\lambda}
$$

where $\left\{{ }_{\mu \nu}^{\lambda}\right\}$ is the usual Christoffel symbol and the rest is a combination of torsion and nonmetricity tensors defined as follows:

$$
\begin{gathered}
K_{\mu \nu}^{\lambda}=g^{\sigma \lambda}\left(S_{\mu \sigma \nu}+S_{\nu \sigma \mu}+S_{\mu \nu \sigma}\right), \\
L_{\mu \nu}^{\lambda}=\frac{1}{2} g^{\sigma \lambda}\left(-Q_{\mu \nu \sigma}-Q_{\nu \mu \sigma}+Q_{\sigma \mu \nu}\right) .
\end{gathered}
$$

Accordingly, the affine curvature tensor will take the form of

$$
\begin{aligned}
\mathbf{R}_{\beta \mu \nu}^{\lambda}= & R_{\beta \mu \nu}^{\lambda}(\{\})-\nabla_{\{v\}} L_{\beta \mu}^{\lambda}+\nabla_{\{\mu\}} L_{\beta \nu}^{\lambda} \\
& +L_{\alpha \mu}^{\lambda} L_{\beta \nu}^{\alpha}-L_{\alpha \nu}^{\lambda} L_{\beta \mu}^{\alpha}-\nabla_{\{\nu\}} K_{\beta \mu}^{\lambda}+\nabla_{\{\mu\}} K_{\beta \nu}^{\lambda} \\
& +K_{\alpha \mu}^{\lambda} K_{\beta \nu}^{\alpha}-K_{\alpha \nu}^{\lambda} K_{\beta \mu}^{\alpha}+L_{\alpha \mu}^{\lambda} K_{\beta \nu}^{\alpha} \\
& +L_{\beta \nu}^{\alpha} K_{\alpha \mu}^{\lambda}-L_{\beta \mu}^{\alpha} K_{\alpha \nu}^{\lambda}-L_{\alpha \nu}^{\lambda} K_{\beta \mu}^{\alpha},
\end{aligned}
$$

where $R_{\beta \mu \nu}^{\lambda}(\{\})$ indicates Riemann curvature tensor and braces are used to show covariant derivative in Riemann geometry. Contraction of (8) will result in two different ranktwo tensors $[4,23]$; one of them is the affine Ricci tensor obtained by contracting the first index with the last pair of indices $\left(\mathbf{R}_{\beta v} \equiv \mathbf{R}_{\beta \lambda v}^{\lambda}\right)$ and the other one is the so-called homothetic curvature tensor which resulted from contraction of the first two indices $\left(\widehat{\mathbf{R}}_{\mu \nu} \equiv \widehat{\mathbf{R}}_{\lambda \mu \nu}^{\lambda}\right)$. Generation of the two second rank curvature tensors is due to the fact that the only symmetry of the affine curvature tensor is the antisymmetric property of the last pair of indices. However, there is just one independent affine Ricci scalar and the contraction of homothetic curvature tensor with metric gives rise to vanishing scalar because of its antisymmetric nature $[4,23]$. Having more complete description of geometry, in the following section, we will focus on constructing the generic gravitational action based on metric-affine geometry. 


\section{Metric-Affine Second Order Action}

With independence of the metric and connection in MAG, the general gravitational action is expressed in terms of metric and connection and their derivatives. Also the coupling of matter action with connection in addition to metric and matter fields is allowed in the case of MAG which forms the dissimilarity between this approach and the Palatini method $[23,27]$. In this paper, we limit our discussion to the geometrical part of the action, but what the form of the action is. Obviously, the first choice is to replace the Einstein-Hilbert $(\mathrm{EH})$ action with its counterpart in metric-affine formalism, but this is not the only possibility and extension of $\mathrm{EH}$ action into a more general one through the metric-affine formalism can be done by considering torsion and nonmetricity tensors as well as curvature tensor in construction of the action. (In our study, only the geometrical part of the action is dealt with, so the inconsistency that is mentioned in $[28,29]$ does not appear in our consideration.) In order to construct the gravitational Lagrangian, we follow the approach of $[23,30]$ which is an effective field theory approach and appropriate scalar terms are constructed at each order by applying power counting analysis. To start with and in natural units $c=\hbar=1$, by choosing $[d x]=[d t]=[l]$, all of the geometrical quantities, which are needed in construction of the action, are expressed in terms of length dimension and, consequently, to make the action dimensionless, the coupling constant is related to Planck length $l_{p}$. The highest power of the length dimension in the scalars shows the order of action so the first term of the generalized action, which is the Ricci scalar that is replaced by the affine one, is of second order.

Restricting our discussion to second order action and considering symmetries of torsion and nonmetricity tensors, there will be four scalars written in terms of torsion tensor and its derivative, eight terms made up of the nonmetricity tensor and its derivative, and three terms constructed from the contraction of torsion and nonmetricity tensors. (Terms of higher orders have been presented in [31] and, for a less general case, in [23].) Accordingly, the generic second order gravitational action in $n$ dimensions takes the following form: (a similar action has been studied in [31] with different approach in the context of scalar, vector, tensor theory. Note also that it is proved that, due to the symmetry properties, the other possible forms of nonvanishing scalars can be rewritten using the terms of this action)

$$
\begin{aligned}
& \mathscr{I}_{\text {MAG }} \\
& =\frac{1}{\kappa} \int d^{n} x \sqrt{-g}\left(a_{0} \mathbf{R}+a_{1} \nabla^{\mu} S_{\mu}\right. \\
& +a_{2} S^{\mu} S_{\mu}+a_{3} S_{\lambda}^{\mu}{ }^{\sigma} S_{\mu \sigma}{ }^{\lambda}+a_{4} S^{\mu \nu \lambda} S_{\mu \nu \lambda} \\
& +a_{5} \nabla^{\mu} Q_{\mu \sigma}^{\sigma}+a_{6} g^{\nu \sigma} \nabla_{\mu} Q^{\mu}{ }_{\nu \sigma} \\
& +a_{7} g^{\mu \nu} Q^{\lambda}{ }_{\mu \lambda} Q^{\sigma}{ }_{\nu \sigma}+a_{8} g^{\mu \lambda} Q^{\nu}{ }_{\mu \lambda} Q^{\sigma}{ }_{\nu \sigma} \\
& +a_{9} g^{\mu \nu} Q^{\sigma}{ }_{\mu \lambda} Q^{\lambda}{ }_{\nu \sigma} \\
& +a_{10} g^{\mu \alpha} g^{\nu \beta} g_{\lambda \gamma} Q_{\mu \nu}^{\lambda} Q_{\alpha \beta}^{\gamma}
\end{aligned}
$$

$$
\begin{aligned}
& +a_{11} g^{\mu \nu} g^{\alpha \beta} g_{\lambda \gamma} Q_{\mu \nu}^{\lambda} Q_{\alpha \beta}^{\gamma} \\
& +a_{12} g^{\mu \nu} Q_{\mu \nu}^{\lambda} S_{\lambda}+a_{13} g^{\mu \nu} Q_{\mu \lambda}^{\lambda} S_{\nu} \\
& \left.+a_{14} g^{\mu \alpha} Q_{\mu \nu}^{\lambda} S_{\lambda \alpha}{ }^{\nu}\right)
\end{aligned}
$$

in which $S_{\mu} \equiv S_{\mu \beta}{ }^{\beta}$ and $a_{i}$ 's denote different coupling constants, $g$ stands for the determinant of metric, and $\kappa$ contains the Planck length, for example, in four dimensions $\kappa=16 \pi l_{p}^{2}$. The relevant field equations can be obtained by varying the action with respect to metric and connection independently, and then one obtains after a trivial but rather lengthy calculation the field equations which are given in the Appendix. In the following section, we are going to fix the free parameters which appear in the action due to its general form of definition.

\section{Conformal Invariance of the Action}

The action in the form of (9) has free parameters that must be fixed. This can be done by imposing some constraints or initial conditions where we use the conformal invariance condition. The first extension of Einstein's gravity was done by Weyl in 1919 and then developed by Cartan and Dirac (for review see [2, 3, 32, 33]). In [19], conformal invariance was considered in Riemann-Cartan geometry where the torsion plays the role of an effective Weyl gauge field. Conformal torsion gravity and conformal symmetry in teleparallelism were studied in [34].

Under the conformal transformation of metric of the form

$$
\bar{g}_{\mu \nu}(x)=\Omega^{2} g_{\mu \nu}(x),
$$

the nonmetricity, which is associated with $\nabla$, transforms as

$$
\bar{Q}_{\mu \nu}^{\lambda}=2 g_{\mu \nu} \nabla^{\lambda} \ln \Omega+Q_{\mu \nu}^{\lambda},
$$

where $\Omega$ is a scalar function of $x$. However, the conformal transformation of the torsion tensor is somehow ambiguous and different forms of transformation are considered, namely, weak conformal transformation under which the torsion tensor remains unchanged and strong conformal transformation in which the torsion tensor transforms similar to (10); namely, one has $\bar{S}_{\mu \nu}^{\lambda}=\Omega^{2} S_{\mu \nu}{ }^{\lambda}$ [17]. Being interested in conformal invariance of the action, we choose the weak form which preserves the antisymmetric part of the affine connection. In accordance with all what is mentioned above, one can easily show that the affine connection is not changed under the conformal transformation of the form (10).

It is straightforward to show that, under conformal transformation, the action (9) transforms in the following way:

$$
\begin{aligned}
\overline{\mathscr{I}}_{\mathrm{MAG}}= & \Omega^{n-2} \mathscr{I}_{\mathrm{MAG}}+\frac{1}{\kappa} \\
& \times \int d^{n} x \Omega^{n-2}
\end{aligned}
$$




$$
\begin{gathered}
\times \sqrt{-g}\left(\left[2 a_{5}+2 n a_{6}\right] \nabla^{2} \ln \Omega\right. \\
+\left[4 a_{7}+4 n a_{8}+4 a_{9}+4 n a_{10}\right. \\
\left.\quad+4 n^{2} a_{11}\right]\left(\nabla^{\lambda} \ln \Omega\right)\left(\nabla_{\lambda} \ln \Omega\right) \\
+\left[-a_{5}-(n-2) a_{6}+2 a_{8}+4 a_{10}\right. \\
\left.\quad+4 n a_{11}\right] g^{\mu \nu} Q_{\mu \nu}^{\lambda} \nabla_{\lambda} \ln \Omega \\
+\left[2 a_{5}+4 a_{7}+2 n a_{8}\right. \\
\left.+4 a_{9}\right] Q_{\mu \lambda}^{\mu} \nabla^{\lambda} \ln \Omega \\
+\left[4 a_{5}+4 n a_{6}+2 n a_{12}+2 a_{13}\right. \\
\left.\left.+2 a_{14}\right] S_{\lambda} \nabla^{\lambda} \ln \Omega\right)
\end{gathered}
$$

in which $\nabla^{2} \ln \Omega=\nabla_{\{\lambda\}} \nabla^{\{\lambda\}} \ln \Omega$. The conformal invariance of the action results in vanishing of the additional terms at the above relation, which leads one to write

$$
\begin{aligned}
& a_{6}=-\frac{a_{5}}{n}, \\
& a_{9}=-\frac{a_{5}}{2}-a_{7}-\frac{n a_{8}}{2}, \\
& a_{11}=\frac{a_{5}}{2 n^{2}}-\frac{a_{8}}{2 n}-\frac{a_{10}}{n}, \\
& a_{14}=-n a_{12}-a_{13} .
\end{aligned}
$$

Inserting (13) in (9) leads to

$$
\begin{aligned}
& \mathscr{I}_{\mathrm{WMAG}}=\frac{1}{\kappa} \\
& \times \int d^{n} x \sqrt{-g}\left(a_{0} \mathbf{R}+a_{1} \nabla^{\mu} S_{\mu}+a_{2} S_{\mu} S^{\mu}\right. \\
& +a_{3} g^{\mu \nu} S_{\mu \lambda}^{\sigma} S_{\nu \sigma}^{\lambda}+a_{4} S_{\mu \nu \lambda} S^{\mu \nu \lambda} \\
& +a_{5}\left[g^{\mu \nu} \nabla_{\mu} Q_{\nu \sigma}^{\sigma}-\frac{1}{n} g^{\nu \sigma} \nabla_{\mu} Q_{\nu \sigma}^{\mu}\right. \\
& -\frac{1}{2} g^{\mu \nu} Q_{\mu \lambda}^{\sigma} Q_{\nu \sigma}^{\lambda} \\
& \left.+\frac{1}{2 n^{2}} g^{\mu \nu} g^{\alpha \beta} g_{\lambda \gamma} Q_{\mu \nu}^{\lambda} Q_{\alpha \beta}^{\gamma}\right] \\
& +a_{7}\left[g^{\mu \nu} Q_{\mu \lambda}^{\lambda} Q_{\nu \sigma}^{\sigma}\right. \\
& \left.-g^{\mu \nu} Q_{\mu \lambda}^{\sigma} Q^{\lambda}{ }_{\nu \sigma}\right] \\
& +a_{8}\left[g^{\mu \lambda} Q_{\mu \lambda}^{\nu} Q^{\sigma}{ }_{\nu \sigma}\right. \\
& -\frac{n}{2} g^{\mu \nu} Q_{\mu \lambda}^{\sigma} Q^{\lambda}{ }_{\nu \sigma} \\
& \left.-\frac{1}{2 n} g^{\mu \nu} g^{\alpha \beta} g_{\lambda \gamma} Q_{\mu \nu}^{\lambda} Q_{\alpha \beta}^{\gamma}\right]
\end{aligned}
$$

$$
\begin{gathered}
+a_{10}\left[g^{\mu \alpha} g^{\nu \beta} g_{\lambda \gamma} Q_{\mu \nu}^{\lambda} Q_{\alpha \beta}^{\gamma}\right. \\
-\frac{1}{n} g^{\mu \nu} g^{\alpha \beta} g_{\lambda \gamma} Q_{\mu \nu}^{\lambda} Q_{\alpha \beta}^{\gamma}{ }_{\alpha \beta}{ }^{\mu \nu} g_{12}^{\mu \nu} Q_{\mu \nu}^{\lambda} S_{\lambda} \\
\left.-n g^{\mu \alpha} Q_{\mu \nu}^{\lambda} S_{\lambda \alpha}{ }^{\nu}\right] \\
+a_{13}\left[g^{\mu \nu} Q_{\mu \lambda}^{\lambda} S_{\nu}\right. \\
\left.\left.-g^{\mu \alpha} Q_{\mu \nu}^{\lambda} S_{\lambda \alpha}{ }^{\nu}\right]\right) .
\end{gathered}
$$

This is the general form of the Weyl-invariant metric-affine gravity (WMAG), noting that a compensating Weyl scalar is needed to cancel out the $\Omega^{n-2}$ factor which appears in (12).

More simplification can be done if one is interested in the reduced form of metric-affine space; for example, in EinsteinWeyl-Cartan space, one has $\nabla_{\lambda} g_{\mu \nu}=-2 A_{\lambda} g_{\mu \nu}$, where $A_{\lambda}$ is a vector field [22]. By applying this condition to (14), it turns to the following simple form:

$$
\begin{aligned}
\mathscr{I}_{\mathrm{WMAG}}= & \frac{1}{\kappa} \\
\times & \times \int d^{n} x \sqrt{-g}\left(a_{0} \mathbf{R}+a_{1} \nabla^{\mu} S_{\mu}+a_{2} S_{\mu} S^{\mu}\right. \\
& \left.\quad+a_{3} g^{\mu \nu} S_{\mu \lambda}{ }^{\sigma} S_{\nu \sigma}{ }^{\lambda}+a_{4} S_{\mu \nu \lambda} S^{\mu \nu \lambda}\right) .
\end{aligned}
$$

We would like to mention that (15) is expressible in a special form when the affine curvature scalar and covariant derivative are redefined in terms of the Riemannian part plus a particular combination of torsion and nonmetricity squareterms by using (6), (7), and (8). Therefore, the affine curvature scalar takes the form of

$$
\begin{aligned}
\mathbf{R}= & R(\{\})-4 g^{\mu \nu} \nabla_{\{\mu\}} S_{\nu}-4 S_{\mu} S^{\mu}+2 g^{\mu \nu} S_{\mu \lambda}{ }^{\sigma} S_{\nu \sigma}{ }^{\lambda} \\
& +S_{\mu \nu \lambda} S^{\mu \nu \lambda}+g^{\nu \sigma} \nabla_{\{\mu\}} Q^{\mu}{ }_{\nu \sigma}-g^{\mu \nu} \nabla_{\{\mu\}} Q_{\nu \sigma}^{\sigma} \\
& +\frac{1}{2} g^{\mu \lambda} Q_{\mu \lambda}^{\nu} Q_{\nu \sigma}^{\sigma}-\frac{1}{2} g^{\mu \nu} Q_{\mu \lambda}^{\sigma} Q_{\nu \sigma}^{\lambda} \\
& +\frac{1}{4} g^{\mu \alpha} g^{\nu \beta} g_{\lambda \gamma} Q_{\mu \nu}^{\lambda} Q_{\alpha \beta}^{\gamma}-\frac{1}{4} g^{\mu \nu} g^{\alpha \beta} g_{\lambda \gamma} Q_{\mu \nu}^{\lambda} Q_{\alpha \beta}^{\gamma} \\
& +2 g^{\mu \nu} Q_{\mu \nu}^{\lambda} S_{\lambda}-2 g^{\mu \nu} Q_{\mu \lambda}^{\lambda} S_{\nu}-2 g^{\mu \alpha} Q_{\mu \nu}^{\lambda} S_{\lambda \alpha}{ }^{\nu} .
\end{aligned}
$$

And, in Einstein-Weyl-Cartan space, it becomes

$$
\begin{aligned}
\mathbf{R}= & R(\{\})-2(n-1) \nabla_{\{\sigma\}} A^{\sigma}-(n-1)(n-2) A^{2} \\
& -4(n-2) A^{\mu} S_{\mu}-4 g^{\mu \nu} \nabla_{\{\mu\}} S_{\nu}-4 S_{\mu} S^{\mu} \\
& +2 g^{\mu \nu} S_{\mu \lambda}{ }^{\sigma} S_{\nu \sigma}^{\lambda}+S_{\mu \nu \lambda} S^{\mu \nu \lambda} .
\end{aligned}
$$

With the same procedure, one obtains

$$
\nabla^{\mu} S_{\mu}=g^{\mu \nu} \nabla_{\{\mu\}} S_{\nu}+2 S^{\mu} S_{\mu}+(n-2) A^{\mu} S_{\mu}
$$


and substituting (17) and (18) in (15) results in

$$
\begin{aligned}
\mathscr{I}_{\mathrm{WMAG}}^{s p .}=\frac{1}{\kappa} & \\
\times \int d^{n} x \sqrt{-g}( & a_{0} R(\{\})-2(n-1) a_{0} \nabla_{\{\sigma\}} A^{\sigma} \\
& -(n-1)(n-2) a_{0} A^{2} \\
& -\left(4 a_{0}-a_{1}\right) g^{\mu \nu} \nabla_{\{\mu\}} S_{\nu} \\
& -\left(4 a_{0}-2 a_{1}-a_{2}\right) S_{\mu} S^{\mu} \\
& +\left(2 a_{0}+a_{3}\right) g^{\mu \nu} S_{\mu \lambda}{ }^{\sigma} S_{\nu \sigma}{ }^{\lambda} \\
& +\left(a_{0}+a_{4}\right) S_{\mu \nu \lambda} S^{\mu \nu \lambda} \\
& \left.-\left(4(n-2) a_{0}-(n-2) a_{1}\right) A^{\mu} S_{\mu}\right) .
\end{aligned}
$$

Now by imposing the torsion-free condition on (19) and setting $a_{0}=1$, up to a compensating Weyl scalar, it becomes similar to what is studied as a conformally invariant extension of Einstein-Hilbert action [35].

It is worth noting that when the action in (14) is transferred to Weitzenböck space by setting the curvature and nonmetricity to zero [13], it reduces to

$$
\begin{aligned}
\mathscr{I}_{\mathrm{WMAG}}= & \frac{1}{\kappa} \\
\times \int d^{n} x \sqrt{-g}( & a_{1} \nabla^{\mu} S_{\mu}+a_{2} S_{\mu} S^{\mu} \\
& \left.\quad+a_{3} g^{\mu \nu} S_{\mu \lambda}{ }^{\sigma} S_{\nu \sigma}^{\lambda}+a_{4} S_{\mu \nu \lambda} S^{\mu \nu \lambda}\right)
\end{aligned}
$$

which is similar to the one used in teleparallel theories of gravity [29, 36]. For example, in [37], from the tetrad and scalar field analysis of torsion, it is shown that such an action with specific coefficients of $a_{1}=0, a_{2}=-1 / 3, a_{3}=1 / 2$, and $a_{4}=1 / 4$ can indeed be a conformally invariant teleparallel action.

\section{Conclusion}

In this paper, we have first studied the general form of second order metric-affine action which is constructed from all the possible forms of 15 scalar terms made up of affine curvature, torsion, and nonmetricity tensors. The Weyl-invariant extension is obtained by imposing conformal invariance as a condition on the action. The resultant action reduces to the conformally invariant teleparallel action in transition to Weitzenböck space [37]. Studying conformally invariant torsion theories is important because, aside from the conformal invariance property, they can address some important issues of theoretical physics (e.g., see [38]). Torsion theories may be viewed as a rank-3 mixed symmetry tensor field. From the spacetime symmetry and group theoretical point of view, it is proved that linear conformal quantum gravity in flat and de Sitter backgrounds should contain such mixed symmetry tensor field of rank-3 [39-41].

Our obtained action (9) and its Weyl-invariant extension cover the theories made up from Riemann curvature equipped with the torsion and nonmetricity, albeit studying the Weyl invariance of such theories needs some modifications; however, as discussed, in our method, the Weyl invariance can only be obtained by fixing the coefficients, which we have considered as a special case.

\section{Appendix}

\section{Field Equations of the General Action}

In this appendix, we obtain the related field equations of (9) in 4 dimensions. At first, let us variate the action with respect to the metric, which leads to

$$
\begin{aligned}
& a_{0}\left\{\mathbf{R}_{(\mu \nu)}-\frac{1}{2} g_{\mu \nu} \mathbf{R}\right\}+a_{1}\left\{\nabla_{(\mu} S_{\nu)}-\frac{1}{2} g_{\mu \nu} \nabla^{\beta} S_{\beta}\right\} \\
& +a_{2}\left\{-\frac{1}{2} g_{\mu \nu} S_{\alpha} S^{\alpha}+S_{\mu} S_{\nu}\right\} \\
& +a_{3}\left\{-\frac{1}{2} g_{\mu \nu} S_{\alpha \lambda \sigma} S^{\alpha \sigma \lambda}+S_{\mu \lambda}{ }^{\sigma} S_{\nu \sigma}^{\lambda}\right\} \\
& +a_{4}\left\{-\frac{1}{2} g_{\mu \nu} S_{\rho \sigma \lambda} S^{\rho \sigma \lambda}+2 S^{\alpha}{ }_{\nu \lambda} S_{\alpha \mu}{ }^{\lambda}-S_{\rho \sigma \mu} S^{\rho \sigma}{ }_{\nu}\right\} \\
& +a_{5}\left\{-\nabla_{\nu} \nabla_{\mu} \ln \sqrt{-g}-\left(\nabla_{\nu} \ln \sqrt{-g}\right)\left(\nabla_{\mu} \ln \sqrt{-g}\right)\right. \\
& +2 Q_{\alpha(\mu}^{\alpha} \nabla_{\nu)} \ln \sqrt{-g}+4 S_{(\mu} \nabla_{\nu)} \ln \sqrt{-g}+g_{\mu \kappa} \nabla_{\nu} Q_{\alpha}{ }^{\alpha \kappa} \\
& +\nabla_{\mu} Q_{\sigma \nu}^{\sigma}-\frac{1}{2} g_{\mu \nu} \nabla^{\beta} Q_{\sigma \beta}^{\sigma}+2 \nabla_{\nu} S_{\mu}+Q_{\rho}{ }^{\rho \gamma} Q_{\nu \gamma \mu} \\
& \left.-Q_{\sigma \nu}^{\sigma} Q^{\kappa}{ }_{\kappa \mu}-4 S_{\mu} S_{\nu}-4 S_{(\mu} Q_{\sigma \mid v)}^{\sigma}\right\} \\
& +a_{6}\left\{-g_{\mu \nu} \nabla_{\beta} \nabla^{\beta} \ln \sqrt{-g}-g_{\mu \nu}\left(\nabla_{\beta} \ln \sqrt{-g}\right)\left(\nabla^{\beta} \ln \sqrt{-g}\right)\right. \\
& -Q_{\nu \sigma}{ }^{\sigma} \nabla_{\mu} \ln \sqrt{-g}+2 Q_{\mu \nu}^{\alpha} \nabla_{\alpha} \ln \sqrt{-g} \\
& +4 g_{\mu \nu} S^{\beta} \nabla_{\beta} \ln \sqrt{-g}+2 \nabla_{\alpha} Q_{\mu \nu}^{\alpha} \\
& -\frac{1}{2} g_{\mu \nu} g^{\sigma \beta} \nabla_{\alpha} Q_{\sigma \beta}^{\alpha}+2 g_{\mu \nu} \nabla_{\beta} S^{\beta}+Q_{\mu}^{\sigma \rho} Q_{\nu \sigma \rho} \\
& \left.-2 Q^{\alpha \lambda}{ }_{\nu} Q_{\alpha \lambda \mu}-4 g_{\mu \nu} S^{\alpha} S_{\alpha}+2 S_{\mu} Q_{\nu \sigma}{ }^{\sigma}-4 S_{\alpha} Q^{\alpha}{ }_{\mu \nu}\right\} \\
& +a_{7}\left\{2 Q_{\beta \mu}^{\beta} \nabla_{\nu} \ln \sqrt{-g}+2 \nabla_{\nu} Q_{\beta \mu}^{\beta}-\frac{1}{2} g_{\mu \nu} Q_{\lambda \alpha}^{\lambda} Q_{\sigma}{ }^{\sigma \alpha}\right. \\
& \left.-Q_{\beta \mu}^{\beta} Q_{\lambda \nu}^{\lambda}-4 S_{\nu} Q_{\beta \mu}^{\beta}\right\} \\
& +a_{8}\left\{Q_{\mu \beta}{ }^{\beta} \nabla_{\nu} \ln \sqrt{-g}+g_{\mu \nu} Q_{\beta}{ }^{\beta \sigma} \nabla_{\sigma} \ln \sqrt{-g}+g_{\mu \nu} \nabla^{\kappa} Q_{\beta \kappa}^{\beta}\right. \\
& +g^{\beta \lambda} \nabla_{\nu} Q_{\mu \beta \lambda}+2 Q_{\sigma[\mu}^{\sigma} Q_{\nu] \beta}{ }^{\beta}-Q_{\nu}{ }^{\beta \lambda} Q_{\mu \beta \lambda} \\
& -g_{\mu \nu} Q_{\sigma}{ }^{\sigma \kappa} Q_{\beta \kappa}^{\beta}-\frac{1}{2} g_{\mu \nu} Q_{\lambda}^{\beta \lambda} Q_{\sigma \beta}^{\sigma} \\
& \left.-2 S_{\nu} Q_{\mu \sigma}{ }^{\sigma}-2 g_{\mu \nu} S^{\rho} Q_{\beta \rho}^{\beta}\right\}
\end{aligned}
$$




$$
\begin{aligned}
& +a_{9}\left\{2 Q_{\nu \mu \sigma} \nabla^{\sigma} \ln \sqrt{-g}-2 Q_{\nu \mu \sigma} Q_{\beta}^{\beta \sigma}+2 \nabla^{\sigma} Q_{\nu \mu \sigma}\right. \\
& \left.-\frac{1}{2} g_{\mu \nu} Q^{\sigma \beta \lambda} Q_{\lambda \beta \sigma}-Q_{\mu \sigma}^{\lambda} Q_{\lambda \nu}^{\sigma}-4 S^{\sigma} Q_{\nu \mu \sigma}\right\} \\
& +a_{10}\left\{2 Q_{\mu \nu}^{\sigma} \nabla_{\sigma} \ln \sqrt{-g}+2 \nabla_{\sigma} Q_{\mu \nu}^{\sigma}-\frac{1}{2} g_{\mu \nu} Q^{\lambda \alpha \beta} Q_{\lambda \alpha \beta}\right. \\
& \left.+Q_{\nu}{ }^{\alpha \beta} Q_{\mu \alpha \beta}-2 Q^{\sigma \alpha}{ }_{\nu} Q_{\sigma \alpha \mu}-4 S_{\sigma} Q_{\mu \nu}^{\sigma}\right\} \\
& +a_{11}\left\{2 g_{\mu \nu} Q_{\sigma \beta}{ }^{\beta} \nabla^{\sigma} \ln \sqrt{-g}+2 g_{\mu \nu} g^{\alpha \beta} \nabla_{\sigma} Q_{\alpha \beta}^{\sigma}\right. \\
& -\frac{1}{2} g_{\mu \nu} Q^{\lambda \sigma}{ }_{\sigma} Q_{\lambda \alpha}{ }^{\alpha}+Q_{\mu \alpha}{ }^{\alpha} Q_{\nu \rho}{ }^{\rho} \\
& \left.-2 g_{\mu \nu} Q^{\sigma \alpha \beta} Q_{\sigma \alpha \beta}-4 g_{\mu \nu} S^{\sigma} Q_{\sigma \beta}^{\beta}\right\} \\
& +a_{12}\left\{g_{\mu \nu} S^{\beta} \nabla_{\beta} \ln \sqrt{-g}+g_{\mu \nu} \nabla_{\beta} S^{\beta}-2 g_{\mu \nu} S^{\lambda} S_{\lambda}\right. \\
& \left.-\frac{1}{2} g_{\mu \nu} S^{\lambda} Q_{\lambda \beta}{ }^{\beta}+S_{\mu} Q_{\nu \alpha}{ }^{\alpha}\right\} \\
& +a_{13}\left\{S_{\mu} \nabla_{\nu} \ln \sqrt{-g}+\nabla_{\nu} S_{\mu}-2 S_{\nu} S_{\mu}\right. \\
& \left.-\frac{1}{2} g_{\mu \nu} S^{\alpha} Q_{\lambda \alpha}^{\lambda}+2 Q_{\lambda[\mu}^{\lambda} S_{\nu]}\right\} \\
& +a_{14}\left\{S_{\lambda \mu \nu} \nabla^{\lambda} \ln \sqrt{-g}+g_{\rho \nu} \nabla^{\lambda} S_{\lambda \mu}{ }^{\rho}-2 S^{\lambda} S_{\lambda \mu \nu}\right. \\
& -\frac{1}{2} g_{\mu \nu} S^{\lambda \beta \rho} Q_{\lambda \beta \rho}-S_{\lambda \alpha \nu} Q_{\mu}^{\lambda \alpha}+S_{\mu}{ }^{\beta \rho} Q_{\nu \beta \rho} \\
& \left.-S_{\lambda \mu \nu} Q_{\sigma}{ }^{\sigma \lambda}+S_{\lambda \nu}{ }^{\beta} Q^{\lambda}{ }_{\mu \beta}\right\}=-\frac{\kappa}{\sqrt{-g}} \frac{\delta \mathscr{I}_{M}}{\delta g^{\mu \nu}},
\end{aligned}
$$

where $\mathscr{I}_{M}$ stands for matter action.

Variation of the action with respect to the connection yields the following equation:

$$
\begin{aligned}
& a_{0}\left\{-g^{\mu \nu} \nabla_{\lambda} \ln \sqrt{-g}+\delta^{\nu}{ }_{\lambda} \nabla^{\mu} \ln \sqrt{-g}+Q_{\lambda}{ }^{\mu \nu}\right. \\
& \left.-Q_{\sigma}{ }^{\sigma \mu} \delta_{\lambda}^{\nu}-2 S_{\lambda}^{\mu \nu}+2 g^{\mu \nu} S_{\lambda}-2 S^{\mu} \delta_{\lambda}^{\nu}\right\} \\
& +a_{1}\left\{\delta_{\lambda}^{[\mu} \nabla^{\nu]} \ln \sqrt{-g}+Q_{\alpha}{ }^{\alpha[\mu} \delta_{\lambda}^{\nu]}-g^{\mu \nu} S_{\lambda}+2 S^{[\mu} \delta_{\lambda}^{\nu]}\right\} \\
& +a_{2}\left\{2 S^{[\mu} \delta_{\lambda}^{\nu]}\right\}+a_{3}\left\{2 S_{\lambda}^{[\nu \mu]}\right\}+a_{4}\left\{2 S_{\lambda}^{[\mu \nu]}\right\} \\
& +a_{5}\left\{g^{\mu \nu} \nabla_{\lambda} \ln \sqrt{-g}+\delta_{\lambda}^{\nu} \nabla^{\mu} \ln \sqrt{-g}-2 g^{\mu \nu} Q_{\gamma \lambda}^{\gamma}\right. \\
& \left.-Q_{\rho}^{\rho \mu} \delta_{\lambda}^{\nu}-2 g^{\mu \nu} S_{\lambda}-2 \delta_{\lambda}^{\nu} S^{\mu}\right\} \\
& +a_{6}\left\{2 \delta_{\lambda}^{\mu} \nabla^{\nu} \ln \sqrt{-g}-4 Q_{\lambda}^{\nu \mu}+Q_{\gamma}^{\mu \gamma} \delta_{\lambda}^{\nu}-4 S^{\nu} \delta_{\lambda}^{\mu}\right\} \\
& +a_{7}\left\{-2 g^{\mu \nu} Q_{\beta \lambda}^{\beta}-2 Q_{\beta}^{\beta \mu} \delta_{\lambda}^{\nu}\right\} \\
& +a_{8}\left\{-g^{\mu \nu} Q_{\lambda \rho}{ }^{\rho}-2 Q_{\beta}{ }^{\beta \nu} \delta_{\lambda}^{\mu}-Q^{\mu \beta}{ }_{\beta} \delta_{\lambda}^{\nu}\right\} \\
& +a_{9}\left\{-2 Q_{\lambda}^{\mu \nu}-2 Q_{\lambda}{ }^{\mu \nu}\right\}+a_{10}\left\{-4 Q_{\lambda}^{\nu \mu}\right\}
\end{aligned}
$$

$$
\begin{aligned}
& +a_{11}\left\{-4 Q_{\beta}^{\nu \beta} \delta_{\lambda}^{\mu}\right\}+a_{12}\left\{\delta_{\lambda}^{[\nu} Q_{\beta}^{\mu] \beta}-2 \delta_{\lambda}^{\mu} S^{\nu}\right\} \\
& +a_{13}\left\{Q_{\alpha}^{\alpha[\mu} \delta_{\lambda}^{\nu]}-g^{\mu \nu} S_{\lambda}-S^{\mu} \delta_{\lambda}^{\nu}\right\} \\
& +a_{14}\left\{Q_{\lambda}^{[\mu \nu]}+S_{\lambda}^{\mu \nu}+S_{\lambda}^{\nu \mu}\right\}=-\frac{\kappa}{\sqrt{-g}} \frac{\delta \mathscr{I}_{M}}{\delta \Gamma_{\mu \nu}^{\lambda}}
\end{aligned}
$$

\section{Conflict of Interests}

The authors declare that there is no conflict of interests regarding the publication of this paper.

\section{Acknowledgments}

The authors would like to thank M. V. Takook for his helpful guidelines. This work was supported in part by Islamic Azad University, Central Tehran Branch.

\section{References}

[1] S. Capozziello and V. Faraoni, Beyond Einstein Gravity: A Survey of Gravitational Theories for Cosmology and Astrophysics, Springer, New York, NY, USA, 2011.

[2] S. Nojiri and S. D. Odintsov, "Introduction to modified gravity and gravitational alternative for dark energy," International Journal of Geometric Methods in Modern Physics, vol. 4, no. 1, pp. 115-145, 2007.

[3] S. Capozziello and M. De Laurentis, "Extended theories of gravity," Physics Reports, vol. 509, no. 4-5, p. 167, 2011.

[4] H. F. M. Goenner, "On the history of unified field theories," Living Reviews in Relativity, vol. 7, article 2, 2004.

[5] S. Nojiri and S. D. Odintsov, "Unified cosmic history in modified gravity: from $F(R)$ theory to Lorentz non-invariant models," Physics Reports, vol. 505, no. 2-4, pp. 59-144, 2011.

[6] F. W. Hehl, J. D. McCrea, E. W. Mielke, and Y. Ne'eman, "Metric-affine gauge theory of gravity: field equations, Noether identities, world spinors, and breaking of dilation invariance," Physics Reports, vol. 258, no. 1-2, pp. 1-171, 1995.

[7] D. Puetzfeld, "Prospects of non-Riemannian cosmology", in Proceedings of 22nd Texas Symposium on Relativistic Astrophysics at Stanford University, Stanford, Calif, USA, December 2004.

[8] D. Puetzfeld, "Status of non-riemannian cosmology," New Astronomy Reviews, vol. 49, no. 2, pp. 59-64, 2005.

[9] A. V. Minkevich and A. S. Garkun, "Isotropic cosmology in metric-affine gauge theory of gravity," http://arxiv.org/abs/grqc/9805007.

[10] N. J. Poplawski, "Acceleration of the universe in the Einstein frame of a metric-affine $f(R)$ gravity," Classical and Quantum Gravity, vol. 23, no. 6, pp. 2011-2020, 2006.

[11] D. Puetzfeld and Y. N. Obukhov, "Probing non-riemannian spacetime geometry," Physics Letters A, vol. 372, no. 45, pp. 67116716, 2008.

[12] F. W. Hehl and Y. N. Obukhov, "Elie Cartan's torsion in geometry and in field theory, an essay," http://arxiv.org/abs/0711.1535.

[13] M. Yi Mao, A. Tegmark, S. Guth, and S. Cabi, "Constraining torsion with gravity probe B," Physical Review D, vol. 76, no. 10, Article ID 104029, 26 pages, 2007. 
[14] K. S. Stelle, "Renormalization of higher-derivative quantum gravity," Physical Review, D: Particles and Fields: Third Series, vol. 16, no. 4, pp. 953-969, 1977.

[15] P. D. Mannheim and D. Kazanas, "Exact vacuum solution to conformal Weyl gravity and galactic rotation curves," The Astrophysical Journal, vol. 342, no. 2, pp. 635-638, 1989.

[16] P. D. Mannheim, "Conformal cosmology with no cosmological constant," General Relativity and Gravitation, vol. 22, no. 3, pp. 289-298, 1990.

[17] L. Fabbri, "Metric-torsional conformal gravity," Physics Letters $B$, vol. 707, no. 5, pp. 415-417, 2012.

[18] C. N. Karahan, O. Dogangün, and D. A. Demir, "Conformal transformations in metric-affine gravity and ghosts," Annalen der Physik, vol. 524, no. 8, pp. 461-469, 2012.

[19] Y. N. Obukhov, "Conformal invariance and space-time torsion," Physics Letters A, vol. 90, no. 1-2, pp. 13-16, 1982.

[20] J. W. Maluf, "Conformal invariance and torsion in general relativity," General Relativity and Gravitation, vol. 19, no. 1, pp. 57-71, 1987.

[21] I. L. Shapiro, "Physical aspects of the space-time torsion," Physics Reports, vol. 357, no. 2, pp. 113-213, 2002.

[22] T. Moon, J. Lee, and P. Oh, "Conformal invariance in EinsteinCartan-Weyl space," Modern Physics Letters A, vol. 25, no. 37, pp. 3129-3143, 2010.

[23] V. Vitagliano, T. P. Sotiriou, and S. Liberati, "The dynamics of metric-affine gravity," Annals of Physics, vol. 326, no. 5, pp. 12591273, 2011.

[24] T. P. Sotiriou, "Modified actions for gravity: theory and phenomenology," International School for Advanced Studies, 2007, http://arxiv.org/abs/0710.4438.

[25] M. Blau, Lecture Notes on General Relativity, Albert Einstein Center for Fundamental Physics, Bern University, 2012, http://www.blau.itp.unibe.ch/Lecturenotes.html.

[26] A. Kleyn, "Metric-Affine manifold," http://arxiv.org/abs/gr-qc/ 0405028.

[27] T. P. Sotiriou, "The significance of matter coupling in $f(R)$ gravity," in Proceedings of the 11th Marcel Grossmann Meeting on General Relativity, World Scientific, Singapore, 2008.

[28] F. W. Hehl, G. D. Kerlick, and P. von der Heyde, "On a new metric affine theory of gravitation," Physics Letters B, vol. 63, no. 4, pp. 446-448, 1976.

[29] T. Clifton, P. G. Ferreira, A. Padilla, and C. Skordis, "Modified gravity and cosmology," Physics Reports, vol. 513, no. 1-3, pp. 1189, 2012.

[30] V. Vitagliano, "The role of nonmetricity in metric-affine theories of gravity," http://xxx.tau.ac.il/abs/1308.1642vl.

[31] C. N. Karahan, A. Altaş, and D. A. Demir, "Scalars, vectors and tensors from metric-affine gravity," General Relativity and Gravitation, vol. 45, no. 2, pp. 319-343, 2013.

[32] T. Fulton, F. Rohrlich, and L. Witten, "Conformal invariance in physics," Reviews of Modern Physics, vol. 34, no. 3, pp. 442-457, 1962.

[33] L. O'Raifeartaigh, I. Sachs, and C. Wiesendanger, "Weyl gauging and curved space approach to scale and conformal invariance," in Meeting on 70 Years of Quantum Mechanics, Calcutta, India, January-February 1996.

[34] K. Bamba, S. D. Odintsov, and D. Sáez-Gómez, "Conformal symmetry and accelerating cosmology in teleparallel gravity," Physical Review D, vol. 88, no. 8, Article ID 084042, 10 pages, 2013.
[35] M. P. Dabrowski, J. Garecki, and D. B. Blaschke, "Conformal transformations and conformal invariance in gravitation," Annalen der Physik, vol. 18, no. 1, pp. 13-32, 2009.

[36] R. Aldrovandi and J. G. Pereira, "Gravitation: in search of the missing torsion," Annales de la Fondation Louis de Broglie, vol. 32, p. 229, 2007, http://arxiv.org/abs/0801.4148.

[37] J. W. Maluf and F. F. Faria, "Conformally invariant teleparallel theories of gravity," Physical Review D, vol. 85, no. 2, Article ID 027502, 5 pages, 2012.

[38] N. J. Poplawski, "Matter-antimatter asymmetry and dark matter from torsion," Physical Review D-Particles, Fields, Gravitation and Cosmology, vol. 83, no. 8, Article ID 084033, 2011.

[39] B. Binegar, C. Fronsdal, and W. Heidenreich, "Linear conformal quantum gravity," Physical Review D, vol. 27, no. 10, pp. 22492261, 1983.

[40] M. V. Takook and M. R. Tanhayi, "Linear Weyl gravity in de Sitter universe," Journal of High Energy Physics, vol. 2010, article 44, 2010.

[41] M. V. Takook, M. R. Tanhayi, and S. Fatemi, "Conformal linear gravity in de Sitter space," Journal of Mathematical Physics, vol. 51, no. 3, Article ID 032503, 2010. 

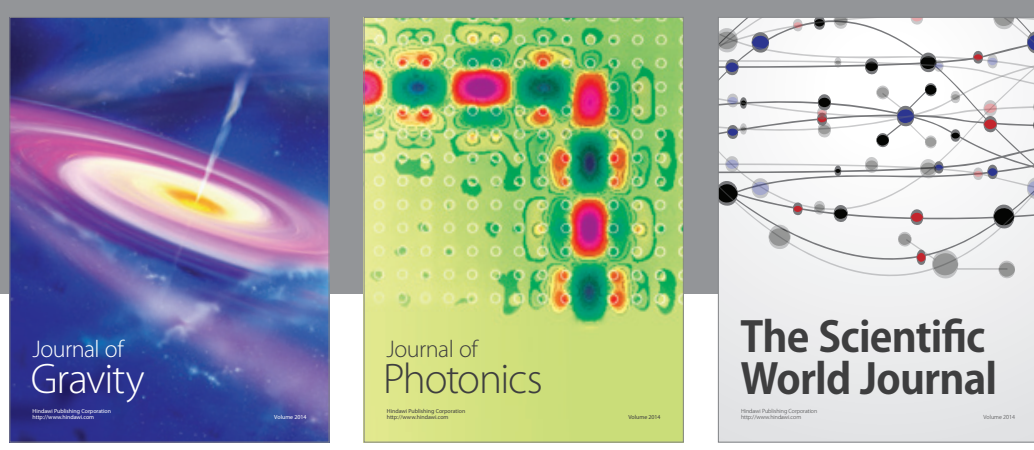

The Scientific World Journal
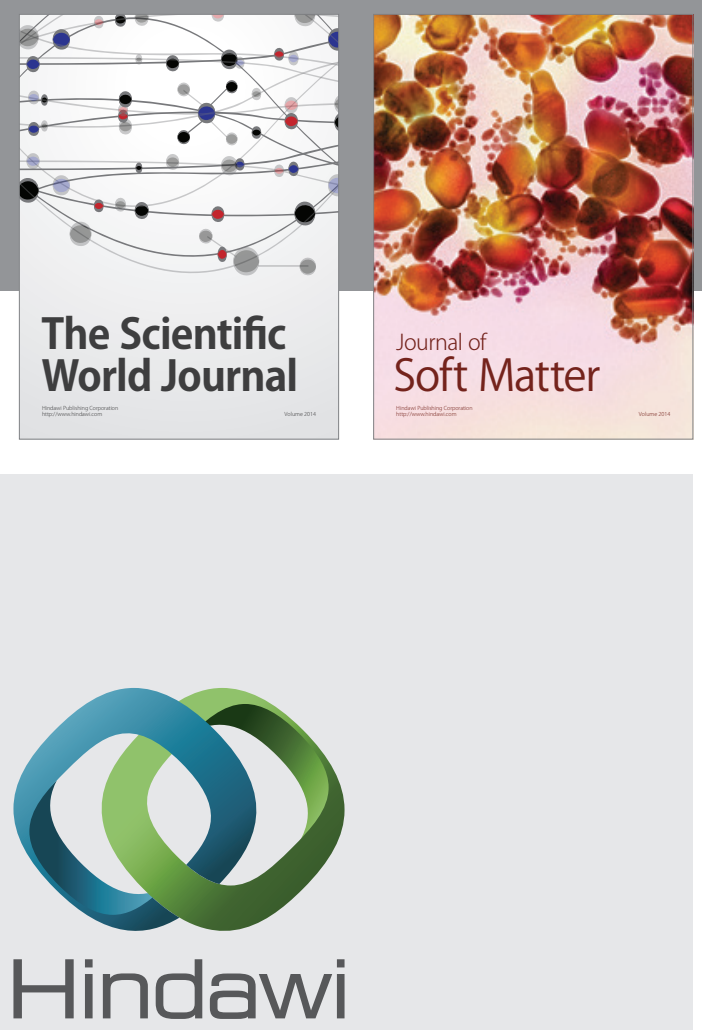

Submit your manuscripts at

http://www.hindawi.com

nternational Journal of

Statistical Mechanics
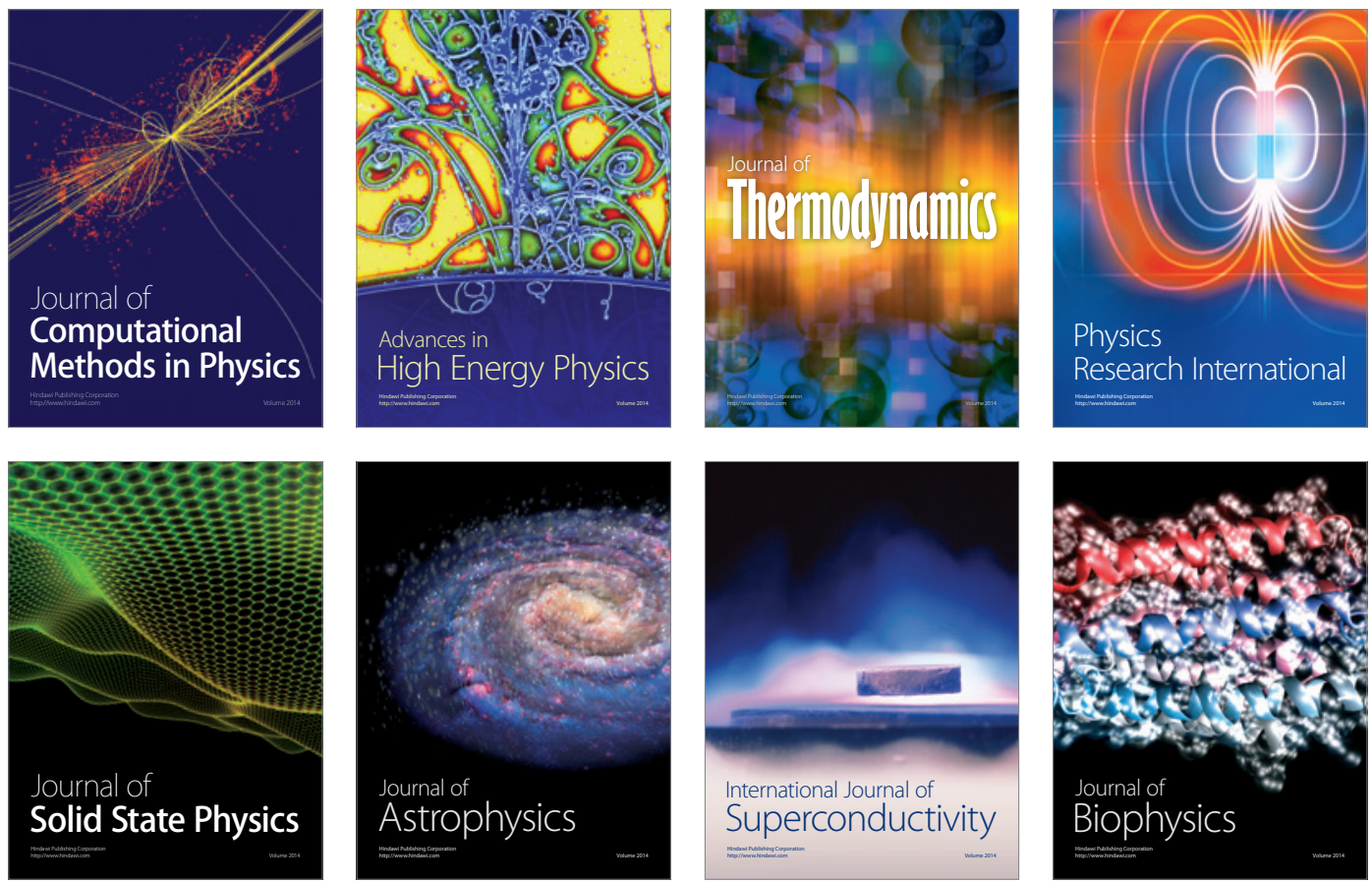
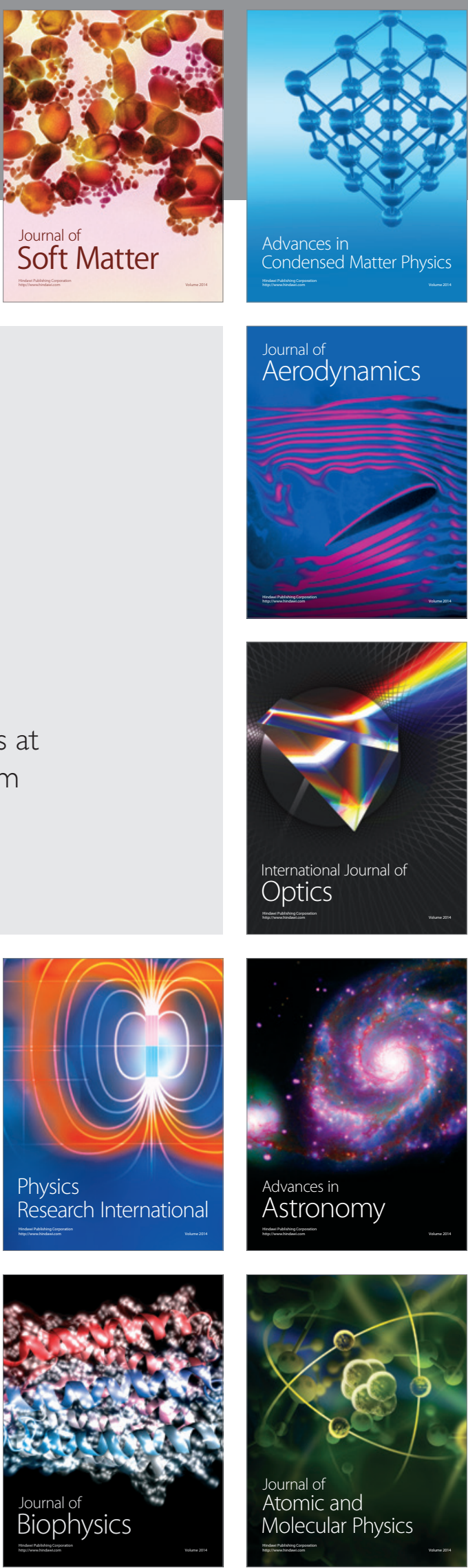